\title{
Generation of motional nonlinear coherent states and their superpositions via intensity-dependent coupling of a cavity field to a micromechanical membrane
}

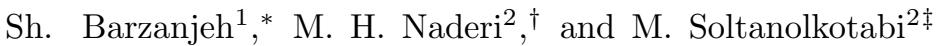 \\ ${ }^{1}$ Department of Physics, Faculty of Science, University of Isfahan, Hezar Jerib, 81746-73441, Isfahan, Iran \\ ${ }^{2}$ Quantum Optics Group, Department of Physics, Faculty of Science, \\ University of Isfahan, Hezar Jerib, 81746-73441, Isfahan, Iran
}

(Dated: April 7, 2021)

\begin{abstract}
We propose a theoretical scheme to show the possibility of generating motional nonlinear coherent states and their superposition for an undamped vibrating micromechanical membrane inside an optical cavity. The scheme is based on an intensity-dependent coupling of the membrane to the radiation pressure field. We show that if the cavity field is initially prepared in a Fock state, the motional state of the membrane may evolve from vacuum state to a special type of nonlinear coherent states. By examining the nonclassical properties of the generated state of the membrane, including the quadrature squeezing and the sub-Poissonian statistics, we find that by varying the Lamb-Dicke parameter and the membrane's reflectivity one can effectively control those properties. In addition, the scheme offers the possibility of generating various types of the so-called nonlinear multicomponent Schrödinger cat sates of the membrane. We also examine the effect of the damping of the cavity field on the motional state of the membrane.
\end{abstract}

PACS numbers: 42.50.Wk, 42.50.Ct, 42.50.Dv

Keywords: nonlinear opto-mechanics, nonlinear coherent states, quantum state engineering.

\section{INTRODUCTION}

The coupling of mechanical motion of mesoscopic or macroscopic mirrors to the electromagnetic degrees of freedom via radiation pressure 1, 2, is a promising approach for applications ranging from the detection of weak forces and small displacements to fundamental studies of the transition between the quantum and the classical world [3]. The canonical optomechanical system consists of an optical cavity where one of the end mirrors is free to move [4. The radiation pressure acting on the movable mirror realizes an optomechanical coupling between the incident optical modes and the various vibrational modes of the mirror. The use of optomechanical coupling has been proposed many years ago for the implementation of quantum limited measurements of mechanical forces [5], as the interferometric detection of gravitational waves [6] or atomic force microscopy [7]. Since then, optomechanical systems have generated much experimental and theoretical interest $8-12]$. They offer the prospect of realizing quantum effects at a macroscopic scale [13], of supplying quantum sensors for applications ranging from single molecule detection [14 to gravitational wave interferometry [15, 16, for the quantum control of atomic, molecular, and optical systems [17, and for possible new quantum information processing devices[18. The optomechanical cooling and trapping of mirrors has also recently become the subject of an intense research effort [9 11, 15, 19] as it offers a viable means of exploring quantum effects such as superposition

\footnotetext{
* shabirbarzanjeh@yahoo.com

$\dagger$ mhnaderi@phys.ui.ac.ir

$\ddagger$ soltan@sci.ui.ac.ir
}

and entanglement at a macroscopic scale 13,20 .

The main technical barrier to reaching the regime in which either the mechanical device itself or its readout demonstrate quantum behavior, has been difficulty of integrating ultrasensitive micromechanical devices with high-finesse optical cavities. Recently, it has been developed a novel type of optomechanical system, the socalled membrane-in-the middle geometry, for addressing this issue in which the mechanical degree of freedom is a flexible, partially transparent dielectric membrane placed inside a Fabry-Perot cavity with fixed end mirrors 21. 23. This has the advantage of not having to combine the flexibility needed for the mechanical oscillator with the rigidity of a high-finesse cavity mirror. Although the membrane is nearly transparent, it couples to the optical cavity dispersively. This coupling is strong enough to laser-cool a 50-nm-thick dielectric membrane from room temperature $(294 \mathrm{~K})$ down to $7 \mathrm{mK}[21]$. In addition, the dispersive nature of the optomechanical coupling allows for realization of a sensitive displacement squared readout of the membrane 21. Such a readout is a crucial requirement for measuring quantum jumps in a mechanical oscillator. It has been shown theoretically 24 that an optomechanical system with the membrane-in-the-middle geometry can be used to detect phonon Fock states. Furthermore, for that system it has been predicted [25] twophonon cooling of the mechanical oscillator, squeezing of the mechanical oscillator, and squeezing of the optical output field. The extension of cavity optomechanics to multimembrane systems has also been considered 26].

In connection with quantum state engineering, optomechanical systems have also attracted considerable attention because of the great possibilities they have to produce nonclassical states of both, the mechanical oscillator and cavity field $[4,8,12,13,20,27,30$. Similar to 
the case of a light field where the imprint of the quantum regime is signaled by generation of nonclassical states, the generation of nonclassical states of a mechanical oscillator can be a hallmark for quantum control of a macroscopic massive object 31. The possibilities to generate nonclassical states, and in particular a superposition of coherent states of the quantized field are because of the Kerr-like Hamiltonian that may be obtained in the optomechanical systems 27, 28. Besides, the fact that the motion of the mechanical oscillator is quantized allows the possibility to generate a large variety of nonclassical states of the mirror [27]. It is shown that the mirror can also be prepared in a Schrödinger cat state with many components by a quadrature measurement of the cavity field after its interaction with the moving mirror. Moreover, the possibility of generating even and odd coherent states of a moving mirror has been considered 32 . A detailed analysis of the effects of finite temperature on creating and verifying a macroscopic quantum superposition in a micro-optomechanical system has been presented in 33. It has been shown that an unambiguous demonstration of a quantum superposition requires the mechanical resonator to be in or near the ground state. In Ref. 8 the authors propose a scheme for transferring quantum states from the propagating light fields to macroscopic, collective vibrational degree of freedom of a massive mirror by exploiting radiation pressure effects. Some of other more recent proposals include the generation of squeezed states of a vibrating membrane or a movable mirror via reservoir engineering in an optomechanical setup 30, the creation of EPR beams in an optomechanical system 34, and the creation of robust entanglement against increasing temperature between the optical intracavity mode and the mechanical mode of the mirror 35 .

In recent years, there has been paid much attention to the study of a family of generalized coherent states, the so-called nonlinear coherent states(NLCSs) 36 because of their relevance in nonlinear quantum optics. They correspond to nonlinear algebras rather than Lie algebras 37. For a detailed discussion of the mathematical properties of NLCSs we refer the reader to Ref. 37, 38. The NLCSs, and their superposition exhibit nonclassical features like amplitude squeezing, subPoissonian statistics and self-splitting accompanied by pronounced quantum interference effects 39. These states are not mere mathematical objects. In the context of ion trap, the microscopic NLCSs may appear as stationary states of the center-of-mass motion of a trapped and laser-driven ion far from the Lamb-Dicke regime 36. The method makes use of the strong nonlinearities inherent in the Jaynes-Cummings Hamiltonian for the laser-assisted vibronic interaction [40. Very recently, it has been introduced 41] a physical scheme that allows one to prepare and control a special kind of these states, the so-called sphere-coherent states, as the motional dark states of a properly laser-driven trapped-atom system. Furthermore, it has been explored 42 the possibility of generating various families of NLCSs in a lossless micromaser within the framework of intensity-dependent Jaynes-Cummings model.

Motivated by the above-mentioned studies on the generation of various types of nonclassical states in an optomechanical structure, in the present paper we deal with the question of how the NLCSs can be generated in an optomechanical system. The system under consideration is an optomechanical cavity with membrane-in-the middle geometry that consists of a high finesse cavity with two perfectly reflecting fixed end mirrors, and a partially reflective movable middle mirror(such as a dielectric membrane) 21 23, 43]. In this type of optomechanical structure, the radiation pressure and cavity detuning are periodic in the membrane displacement. This periodicity leads to an intensity-dependent interaction of the radiation pressure field with the movable membrane. As we shall see, in the absence of any damping mechanisms, this system can lead to the generation of motional nonlinear coherent states and their superposition for the membrane. We also study the nonclassical properties of generated state and show that how the system parameters (in particular, membrane's reflectivity and the Lamb-Dicke parameter) can strongly affect the dynamics of the system under consideration. If those states are to be observed, then very good isolation of the system from the environment is necessary. Although, by taking into account the relevant experimental parameters 21, 22], just the same considered in this paper, one can neglect the membrane's motional damping, but one cannot neglect the cavity damping. Therefore, in order to make the model under consideration more realistic, we also consider the photon leakage from the cavity as a relevant source of decoherence and examine its influence on the motional state of the membrane.

The paper is organized as follows. In Sec. II we first derive an intensity-dependent Hamiltonian describing the coupling of the micromechanical membrane to the radiation pressure field through $j$-phonon excitations of the vibrational sideband. Then, by restricting our treatment to the case of $j=1$ and by using the Feynman disentanglement theorem we derive the time evolution operator of the system. In Sec. III we consider the generation of NLCS of the membrane and investigate the nonclassical properties of the generated state. In Sec. IV the preparation of the nonlinear multicomponent Schrödinger cat states of the membrane is discussed. In Sec. V we take into account the cavity field damping and study its effect on motional state of the membrane. Finally, we summarize our conclusions in Sec. VI. 


\section{THE NONLINEAR COUPLING OF RADIATION PRESSURE FIELD TO A MECHANICAL DEGREE OF FREEDOM}

\section{A. Model Hamiltonian}

As is shown in Fig.1, we consider a high finesse cavity which is detuned by the motion of a partially reflective membrane placed between two macroscopic, rigid, perfectly reflecting fixed end mirrors $21,23,43$. Unlike the standard optomechanical structures, in this type of optomechanical system the coupling between the middle membrane and the optical cavity strongly depends on the position of the membrane. This position dependence results in a cavity detuning, which is a periodic function of the membrane displacement $x$, i.e., $\omega_{c}(x)=(c / L) \cos ^{-1}\left[\left|r_{c}\right| \cos (4 \pi x / \lambda)\right]$ where $L$ and $r_{c}$ are the cavity length and the field reflectivity of the mem- brane, respectively. The Hamiltonian of the system is given by (excluding damping and driving terms) [21, 22]

$$
\hat{H}=\hbar \omega_{c}(\hat{x}) \hat{a}^{\dagger} \hat{a}+\hbar \omega_{m} \hat{b}^{\dagger} \hat{b}
$$

where $\hat{a}$ and $\hat{b}$ are, respectively, the lowering operators for the optical and mechanical modes satisfying the commutation relations $\left[\hat{a}, \hat{a}^{\dagger}\right]=1,\left[\hat{b}, \hat{b}^{\dagger}\right]=1$, and $\omega_{m}$ is the oscillation frequency of the middle membrane. The fact that the motion of the membrane is quantized allows writing the operator of membrane's displacement $\hat{x}$ in terms of the Lamb-Dicke parameter $\eta=\frac{\omega_{p}}{L \omega_{m}} \sqrt{\frac{\hbar}{2 m \omega_{m}}}$,44, 45] $\left(\omega_{p}\right.$ and $m$ denote, respectively, the frequency of the incident field and the motional mass of the membrane)as

$$
\hat{x}=\eta \frac{2 L \omega_{m}}{c}\left(b^{\dagger}+\hat{b}\right) .
$$

By expanding $\omega_{c}(\hat{x})$ in term of $\hat{b}$ and $\hat{b}^{\dagger}$ we obtain

$$
\omega_{c}(\hat{x})=\frac{\pi c}{2 L}-\frac{c}{2 L} \sum_{m=1}^{\infty} \sum_{k=0}^{\frac{m-1}{2}} \frac{\left|r_{c}\right|^{m}}{m}\left(\begin{array}{c}
m \\
k
\end{array}\right) \frac{(m-1) !}{4^{m-1}\left[\left(\frac{m-1}{2}\right) !\right]^{2}}\left\{e^{i \eta \theta(m-2 k)\left(\hat{b}+\hat{b}^{\dagger}\right)}+h . c .\right\}
$$

where $m=2 l+1$ ( $l$ is an integer number) and $\theta=$ $2 L \omega_{m} / c$. By using the Baker-Campbell-Hausdorff theorem in Eq. (3) we may rewrite the Hamiltonian of equation(1) in the form

$$
\hat{H}=\hat{H}_{0}+\hat{H}_{i n t}^{j},
$$

where

$$
\hat{H}_{0}=\hbar \omega_{0} \hat{a}^{\dagger} \hat{a}+\hbar \omega_{m} \hat{b}^{\dagger} \hat{b}
$$

with $\omega_{0}=\pi c /(2 L)$ as the natural frequency of the cavity without middle membrane, describes the free Hamiltonian of the quantized cavity field and the free motion of the mechanical degree of freedom, and

$$
\hat{H}_{i n t}^{j}=\hbar \hat{a}^{\dagger} \hat{a}\left[\chi_{j}^{*} f_{j}\left(\hat{n}_{b}\right) \hat{b}^{j}+\chi_{j}\left(\hat{b}^{\dagger}\right)^{j} f_{j}\left(\hat{n}_{b}\right)\right],(j=0,1,2, . .)
$$

where

$$
\chi_{j}=\frac{c}{2 L}\left(i \frac{4 \pi}{\lambda} \sqrt{\frac{\hbar}{2 m \omega_{m}}}\right)^{j}
$$

and the nonlinearity function $f_{j}\left(\hat{n}_{b}\right)$ is defined by(appendix A)

$$
f_{j}\left(\hat{n}_{b}\right)=\sum_{m=1} \sum_{k=0}^{\frac{m-1}{2}} \frac{\left|r_{c}\right|^{m}}{m}\left(\begin{array}{c}
m \\
k
\end{array}\right) \frac{(m-1) !}{4^{m-1}\left[\left(\frac{m-1}{2}\right) !\right]^{2}}\left\{e^{-\frac{1}{2}(\eta \theta)^{2}(m-2 k)^{2}}\right\} \frac{\hat{n}_{b} !(m-2 k)^{j}}{\left(\hat{n}_{b}+j\right) !} L_{\hat{n}_{b}}^{j}\left[(\eta \theta)^{2}(m-2 k)^{2}\right],
$$

with $\hat{n}_{b}=\hat{b}^{\dagger} \hat{b}$ and $L_{\hat{n}}^{j}$ as the associated Laguerre polynomial, describes a nonlinear coupling of the radiation pressure field with the movable membrane through $j$-phonon excitations of the vibrational sideband.

\section{B. TIME EVOLUTION}

The time evolution operator corresponding to the Hamiltonian (4) can be evaluated easily. For this purpose let us consider the first excitation of the vibrational sideband by choosing $j=1$ in the Hamiltonian (4):

$$
\hat{H}=\hbar \omega_{0} \hat{a}^{\dagger} \hat{a}+\hbar \omega_{m} \hat{b}^{\dagger} \hat{b}+\hbar \hat{a}^{\dagger} \hat{a}\left[\chi^{*} f\left(\hat{n}_{b}\right) \hat{b}+\chi \hat{b}^{\dagger} f\left(\hat{n}_{b}\right)\right]
$$


where we have defined

$$
\begin{aligned}
\chi \equiv \chi_{1} & =i \frac{2 \pi c}{L \lambda} \sqrt{\frac{\hbar}{2 m \omega_{m}}}, \\
f\left(\hat{n}_{b}\right) & \equiv f_{1}\left(\hat{n}_{b}\right)
\end{aligned}
$$

The nonlinearity function $f\left(\hat{n}_{b}\right)$ plays an important role in our treatment since it determines the form of nonlinearity of the intensity-dependent of the coupling between the cavity field and the membrane. We point out that in the limit of very small values of the Lamb-Dicke parameter $\eta$ and for certain values of the membrane reflectivity $r_{c}$ the nonlinearity function $f\left(n_{b}\right)$ reduces to unity. Fig.2(a) shows $f\left(n_{b}\right)$ as a function of $n_{b}$ for $\eta=0.8$ and $r_{c}=0.99$, while Fig.2(b) displays the behavior of $f\left(n_{b}\right)$ versus $n_{b}$ for $\eta=10^{-5}$ and $r_{c}=0.9$. Obviously, for $f\left(n_{b}\right)=1$ the Hamiltonian (9) reduces to the Hamiltonian of the standard opto-mechanical system 27. Therefore, the inherent nonlinearity of the model under consideration can be attributed to the parameters of $\eta$ and $r_{c}$.

In the following, we consider the unitary time evolution operator in the interaction picture, corresponding to the Hamiltonian 9 that reads

$$
\hat{U}_{\text {int }}^{(j=1)}(t)=\hat{T} \exp \left[-\frac{i}{\hbar} \int_{0}^{t} H_{i n t}^{(j=1)}\left(t^{\prime}\right) d t^{\prime}\right],
$$

where $\hat{T}$ indicates the time ordering operator. By substituting Hamiltonian (9) in the interaction picture

$$
\hat{H}_{i n t}^{(j=1)}=\hbar \hat{a}^{\dagger} \hat{a}\left(\chi^{*} f\left(\hat{n}_{b}\right) \hat{b} e^{-i \omega_{m} t}+\chi \hat{b}^{\dagger} f\left(\hat{n}_{b}\right) e^{i \omega_{m} t}\right),
$$

into Eq.11] and using the Feynman operator calculus 46] to disentangling the time-ordered evolution operator(appendix B) we obtain

$$
\begin{array}{r}
\hat{U}_{i n t}^{(j=1)}(\tau)=e^{\beta \mu(\tau) \hat{a}^{\dagger} \hat{a} \hat{B}^{\dagger}} e^{-\beta^{*} \mu^{*}(\tau) \hat{a}^{\dagger} \hat{a} \hat{B}} \times \\
e^{i|\beta|^{2} \lambda(\tau)\left(\hat{a}^{\dagger} \hat{a}\right)^{2} g\left(\hat{n}_{b}\right)} e^{O\left(\beta^{3}, \hat{n}_{b}\right)+\ldots}
\end{array}
$$

where by definition

$$
\tau=\omega_{m} t ; \beta=\chi / \omega_{m} ; \mu(\tau)=1-e^{i \tau} ; \lambda(\tau)=t+i \mu^{*}(\tau),
$$

and

$$
\begin{aligned}
\hat{B} & =f\left(\hat{n}_{b}\right) \hat{b}, \\
g\left(\hat{n}_{b}\right) & =\left(\hat{n}_{b}+1\right) f^{2}\left(\hat{n}_{b}\right)-\hat{n}_{b} f^{2}\left(\hat{n}_{b}-1\right) .
\end{aligned}
$$

Now we make use of an approximation to simplify the unitary operator in Eq. 13. In this approximation we keep terms up to second order in $\beta$. The accuracy of our approximations may be verified by considering the realistic values of $\beta$ based on the experimental values of the relevant parameters 21, 22]. For $\omega_{m} / 2 \pi=10^{5} \mathrm{~Hz}, m=$ $50 \mathrm{pg}, \lambda=532 \mathrm{~nm}$ and $L=0.67 \mathrm{~cm}$, we have $|\chi| \sim 10 \mathrm{kHz}$ which leads to $|\beta| \sim 0.01$. Within this approximation the time evolution operator in Eq. 13 reduces to

$$
\hat{U}_{\text {int }}^{(j=1)}(\tau) \simeq e^{\beta \mu(\tau) \hat{a}^{\dagger} \hat{a} \hat{B}^{\dagger}} e^{-\beta^{*} \mu^{*}(\tau) \hat{a}^{\dagger} \hat{a} \hat{B}} e^{i|\beta|^{2} \lambda(\tau)\left(\hat{a}^{\dagger} \hat{a}\right)^{2} g\left(\hat{n}_{b}\right)} .
$$

Now, we assume that the membrane is initially prepared in a coherent superposition of the phononic Fock states $|\psi(0)\rangle_{m}=\sum_{k} C_{k}|k\rangle_{m}$ and the cavity field is initially in a coherent superposition of the photonic Fock states $|\psi(0)\rangle_{f}=\sum_{n} D_{n}|n\rangle_{f}$. Thus the initial state vector of the system can be written as

$$
|\psi(0)\rangle=\sum_{k, n} C_{k} D_{n}|k\rangle_{m} \otimes|n\rangle_{f}
$$

The time dependent state of the system is obtained by

$$
|\psi(\tau)\rangle=\hat{U}_{\text {int }}^{(j=1)}(\tau)|\psi(0)\rangle
$$

By substituting (16) and (17) into Eq. 18 we obtain

$$
|\psi(\tau)\rangle=\sum_{n, k} C_{k} D_{n} e^{i n^{2} \Theta g(k)} e^{\Lambda_{n} \hat{B}^{\dagger}} e^{-\Lambda_{n}^{*} \hat{B}}|k\rangle_{m} \otimes|n\rangle_{f},
$$

where $\Lambda_{n}=n \beta \mu(\tau)$ and $\Theta=|\beta|^{2} \lambda(\tau)$. Thus, after some rearrangements we obtain

$$
|\psi(\tau)\rangle=\sum_{n, k} C_{k} D_{n} e^{i n^{2} \Theta g(k)}|n\rangle_{f} \otimes\left|\Lambda_{n, k}(\tau)\right\rangle_{m}
$$

where

$$
\begin{aligned}
\left|\Lambda_{n, k}(\tau)\right\rangle_{m}= & f(k-1) ! \sum_{l, l^{\prime}} \frac{\left(\Lambda_{n}\right)^{l}\left(-\Lambda_{n}^{*}\right)^{l^{\prime}}}{l ! l^{\prime} !} \times \\
& \sqrt{\frac{(k+l) !}{\left(k-l^{\prime}\right) !}} \frac{f\left(k+l-l^{\prime}-1\right) !}{\left[f\left(k-l^{\prime}-1\right) !\right]^{2}}\left|k+l-l^{\prime}\right\rangle_{m},
\end{aligned}
$$

with $k \geq l^{\prime}$. As is seen from Eqs. 20 and (21), if the membrane is initially prepared in a vibrational vacuum state, i.e., if $C_{k}=\delta_{k, 0}$ then after a time $\tau=2 \pi$ it returns to its original state. At all times between $\tau=0$ and $\tau=$ $2 \pi$ the membrane state is entangled with the cavity field state. Furthermore, we see from equation (20) that there is an explicit Kerr-like term in the time evolved state, so that physically one might expect the cavity field to have an evolution similar to that in a Kerr-like nonlinearity.

\section{NLCS OF THE MOVABLE MEMBRANE AND ITS NONCLASSICAL PROPERTIES}

\section{A. Generation of NLCS of the membrane}

In this subsection, we consider the generation of NLCS of a macroscopic membrane. For this purpose we turn our attention to the state 20. We assume a simple situation $C_{k}=\delta_{k, 0}$ that is the membrane is initially prepared in its motional ground state. Experimentally, the vacuum state of the membrane is feasible by cooling the membrane to 
its ground state 2123 . Thus, the time dependent state (20) reduces to

$$
|\psi(\tau)\rangle=\sum_{n} D_{n} e^{i n^{2} \Theta g(0)}|n\rangle_{f} \otimes\left|\Lambda_{n}(\tau)\right\rangle_{m},
$$

where $\left|\Lambda_{n}(\tau)\right\rangle_{m} \equiv\left|\Lambda_{n, 0}(\tau)\right\rangle_{m}$. The explicit expression for the state $\left|\Lambda_{n}(\tau)\right\rangle_{m}$ in the number representation is given by

$$
\left|\Lambda_{n}(\tau)\right\rangle_{m}=e^{\Lambda_{n} \hat{B}^{\dagger}}|0\rangle_{m}=\sum_{l}|l\rangle_{m m}\left\langle l \mid \Lambda_{n}(\tau)\right\rangle_{m},
$$

where

$$
{ }_{m}\left\langle l \mid \Lambda_{n}(\tau)\right\rangle_{m}=\aleph \frac{P(l)}{\sqrt{l !}}\left(\Lambda_{n}\right)^{l},
$$

and the normalization constant reads as

$$
\aleph=\left[\sum_{l} \frac{|P(l)|^{2}}{l !}\left|\Lambda_{n}\right|^{2 l}\right]^{-\frac{1}{2}},
$$

with $P(l)=f(l-1) !=\prod_{j=0}^{l-1} f(j), \quad(l>0)$ and $P(0)=1$. The density matrix of the system can be obtained by using Eq. 222,

$$
\begin{aligned}
\rho(\tau) & =|\psi(\tau)\rangle\langle\psi(\tau)| \\
& =\sum_{n, l} D_{n} D_{l}^{*} e^{i\left(n^{2} \Theta-l^{2} \Theta^{*}\right) g(0)}|n\rangle_{f f}\left\langle l|\otimes| \Lambda_{n}(\tau)\right\rangle_{m m}\left\langle\Lambda_{l}(\tau)\right.
\end{aligned}
$$

and the reduced density matrix of the motional state of the membrane reads as

$$
\begin{aligned}
\rho_{m}(\tau) & =\operatorname{Tr}_{f}[\rho(\tau)] \\
& =\sum_{n}\left|D_{n}\right|^{2} e^{i n^{2}\left(\Theta-\Theta^{*}\right) g(0)}\left|\Lambda_{n}(\tau)\right\rangle_{m m}\left\langle\Lambda_{n}(\tau)\right|
\end{aligned}
$$

where $\left|\Lambda_{n}(\tau)\right\rangle_{m}$ can be identified as a family of NLCSs of the membrane at time $\tau$ whose amplitude depends on $n$. In order to get more clear insight to the above result we present the following argument.

In principle, the nonlinear coherent states $|\zeta ; f\rangle$ are defined as the right hand eigenstates of the generalized(deformed) annihilation operator $\hat{B}=f(\hat{n}) \hat{b}[36$, 38 ,

$$
\hat{B}|\zeta ; f\rangle=\zeta|\zeta ; f\rangle
$$

where $f(\hat{n})$ is a reasonably well-behaved real function of the number operator $\hat{n}=\hat{b}^{\dagger} \hat{b}$ and $\zeta$ is an arbitrary complex number. We may seek for an operator $\hat{C}^{\dagger}$ which is conjugate of the operator $\hat{B}$, that is $\left[\hat{B}, \hat{C}^{\dagger}\right]=1$ while their Hermitian conjugates $\hat{B}^{\dagger}$ and $\hat{C}$ satisfy the dual algebra $\left[\hat{C}, \hat{B}^{\dagger}\right]=1$. Thus it is easily found that

$$
\hat{C}=\frac{1}{f(\hat{n})} \hat{b}, \hat{C}^{\dagger}=\hat{b}^{\dagger} \frac{1}{f(\hat{n})} .
$$

Now, let us assume $D_{n}=\delta_{n, k}$ in Eq. 22 , i.e., the cavity field is initially prepared in $|\psi(0)\rangle_{f}=|k\rangle_{f}$. Thus the reduced density matrix of the membrane reads as

$$
\rho_{m}(\tau)=e^{i k^{2}\left(\Theta-\Theta^{*}\right) g(0)}\left|\Lambda_{k}(\tau)\right\rangle_{m m}\left\langle\Lambda_{k}(\tau)\right|,
$$

These quadrature operators show the position and mothentum operators of the mirror, respectively. They obey
the commutation relation

in which the state vector $\left|\Lambda_{k}(\tau)\right\rangle_{m}$ (given by Eq. 23p) is the right-hand eigenstate of the deformed operator $\hat{C}$ given by Eq.29

$$
\hat{C}\left|\Lambda_{k}(\tau)\right\rangle_{m}=\Lambda_{k}(\tau)\left|\Lambda_{k}(\tau)\right\rangle_{m}
$$

In order to investigate the nonclassical behaviour of the generated NLCS, we focus our attention on the temporal evolution of the quadrature squeezing and of the Mandel parameter in the next subsection.

Squeezed states are known by reduced quantum fluctuations in one quadrature of the field at the expense of the increased fluctuations in the other quadrature. In order nonlinear coherent state of Eq.23. we define

$$
\begin{aligned}
& \hat{X}_{1}(\tau) \equiv \frac{1}{2}\left(\hat{b} e^{i \tau}+\hat{b}^{\dagger} e^{-i \tau}\right), \\
& \hat{X}_{2}(\tau) \equiv \frac{1}{2 i}\left(\hat{b} e^{i \tau}-\hat{b}^{\dagger} e^{-i \tau}\right) .
\end{aligned}
$$

$$
\left[\hat{X}_{1}(\tau), \hat{X}_{2}(\tau)\right]=i / 2,
$$

and, consequently, the variances

$$
<\left(\Delta \hat{X}_{j}(\tau)\right)^{2}>\equiv<\left(\hat{X}_{j}(\tau)\right)^{2}>-<\left(\hat{X}_{j}(\tau)\right)>^{2},(j=1,2)
$$

satisfy the uncertainty relation

$$
\left\langle\left(\Delta \hat{X}_{1}(\tau)\right)^{2}\right\rangle\left\langle\left(\Delta \hat{X}_{2}(\tau)\right)^{2}\right\rangle \geq 1 / 16 .
$$

A quantum state of the membrane is said to be squeezed when one of the quadrature components $\hat{X}_{1}$ and $\hat{X}_{2}$ satisfies the relation

$$
\left\langle\left(\Delta \hat{X}_{j}(\tau)\right)^{2}\right\rangle<1 / 4,(j=1 \text { or } 2) .
$$

The degree of squeezing can be measured by the squeezing parameter $S_{j}(j=1,2)$ defined by

$$
S_{j}(\tau) \equiv 4<\left(\Delta \hat{X}_{j}(\tau)\right)^{2}>-1 .
$$

Then, the condition for squeezing in the quadrature component can be simply written as $S_{j}(\tau)<0$. The squeezing parameter $S_{j}(j=1,2)$ can be expressed in terms of the phonon annihilation and creation operators of the membrane as follows

$$
\begin{aligned}
& S_{1}(\tau)=2 A_{1}(\tau)+2 \operatorname{Re}\left[A_{2}(t)\right]-4\left(\operatorname{Re}\left[A_{3}(\tau)\right]\right)^{2}, \\
& S_{2}(\tau)=2 A_{1}(\tau)-2 \operatorname{Re}\left[A_{2}(\tau)\right]-4\left(\operatorname{Im}\left[A_{3}(\tau)\right]\right)^{2},
\end{aligned}
$$

\section{B. Quadrature squeezing of the NLCS of the membrane} to investigate the quadrature squeezing of the generated 
where

$A_{1}(\tau) \equiv<\hat{b}^{\dagger} \hat{b}>, A_{2}(\tau) \equiv<\hat{b^{2}}>e^{2 i \tau}, A_{3}(\tau) \equiv<\hat{b}>e^{i \tau}$.

Now, we study the temporal behaviour of $S_{2}(\tau)$, which gives information on quadrature squeezing of $X_{2}(\tau)$. Numerical results are presented in Fig.3, where we have plotted $S_{2}(\tau)$ versus the scaled time $\tau$ for different values of the parameters $r_{c}$ and $\eta$. As is clear, the quadrature component $X_{2}(\tau)$ exhibits squeezing repeatedly at times $\tau=(2 m+1) \pi,(m=0,1,2, \ldots)$ and the amplitude of squeezing is strongly influenced by the LambDicke parameter $\eta$. Fig.3(a) shows that for $r_{c}=0.9$ the quadrature squeezing only exists for $\eta>0.19$ and the amplitude of squeezing increases by increasing the parameter $\eta$. Furthermore, we find that with the increasing value of membrane's reflectivity, the quadrature squeezing is strengthened and it appears for smaller values of $\eta$. For example, Fig.3(b) shows the quadrature squeezing of $X_{2}(\tau)$ for $r_{c}=0.98$ (this relatively high value of the membrane's reflectivity has recently been considered in Ref.47). As is seen, for $\eta>0.14$ the quadrature component $X_{2}(\tau)$ exhibits squeezing periodically in the course of time evolution.

\section{Mandel-Parameter}

The Mandel-Parameter corresponding to the NLCS of Eq. (23) is obtained as follows

$$
\begin{aligned}
M(\tau) & =\frac{\left\langle\hat{n}_{b}^{2}(\tau)\right\rangle-\left\langle\hat{n}_{b}(\tau)\right\rangle^{2}}{\left\langle\hat{n}_{b}(\tau)\right\rangle}-1 \\
& =\frac{\sum_{k} \frac{\aleph^{2}\left|\Lambda_{n}(\tau)\right|^{2 k} k^{2} P(k)^{2}}{k !}-\left(\sum_{k} \frac{\aleph^{2}\left|\Lambda_{n}(\tau)\right|^{2 k} k P(k)^{2}}{k !}\right)^{2}}{\sum_{k} \frac{\aleph^{2}\left|\Lambda_{n}(\tau)\right|^{2 k} k P(k)^{2}}{k !}}-1 .
\end{aligned}
$$

This parameter vanishes for Poisson distribution, is positive for the super-Poisson distribution, and is negative for the sub-Poisson distribution. Fig.4(a) shows the time evolution of the Mandel parameter with respect to the scaled time $\tau$ for two values of $\eta$ and for $r_{c}=0.9$. As is seen, the generated NLCS for the motional state of the membrane exhibits the sub-Poissonian statistics in the most of time. In addition, the sub-Poissonian characteristic is enhanced by increasing the Lamb-Dicke parameter. Fig.4(b) shows the behaviour of the Mandel parameter for a higher value of the membrane's reflectivity $r_{c}=0.98$. As is clear, the higher values of $r_{c}$ leads to a considerable enhancement of the sub-Poissonian characteristic.

\section{GENERATION OF SUPERPOSITION OF THE MEMBRANE'S NLCSs}

\section{A. Superposition of NLCSs with different amplitudes}

The system under consideration may also be used to generate a superposition of separated NLCSs of the membrane. In order to verify this claim, we assume that the cavity field is initially prepared in a coherent state, i.e., $D_{n}=e^{-\frac{|\alpha|^{2}}{2}} \frac{\alpha^{n}}{\sqrt{n !}}$. Thus the reduced density matrix of the membrane, Eq.227), takes the following form

$\rho_{m}(\tau)=e^{-|\alpha|^{2}} \sum_{n} \frac{|\alpha|^{2 n}}{n !} e^{i n^{2}\left(\Theta-\Theta^{*}\right) g(0)}\left|\Lambda_{n}(\tau)\right\rangle_{m m}\left\langle\Lambda_{n}(\tau)\right|$.

This state shows a superposition of NLCSs with different amplitudes $\Lambda_{n}$ for each value of $n$. The $Q$-function of the motional state associated with the state 41 , is given by

$$
Q(\tau) \equiv \frac{Q_{m}\left(\gamma_{r}, \gamma_{i}\right)}{\aleph^{2}}=\frac{1}{\pi \aleph^{2}}\left\langle\gamma\left|\rho_{m}(\tau)\right| \gamma\right\rangle=\frac{1}{\pi} e^{-|\alpha|^{2}-|\gamma|^{2}} \sum_{n} q_{n}
$$

where

$$
q_{n}=\frac{|\alpha|^{2 n}}{n !} e^{i n^{2} \Theta g(0)-i n^{2} \Theta^{*} g(0)}\left|1+\sum_{l=1} \frac{\left(\Lambda_{n}|\gamma| e^{i \phi}\right)^{l}}{l !}\right|^{2} .
$$

Fig.5 illustrates the normalized $Q$-function $(Q(\tau))$ for different values of the Lamb-Dicke parameter and the membrane's reflectivity at time $\tau=2.9$ and for $|\alpha|^{2}=4$. As is seen from these figures the separation between nonlinear coherent components is increased by increasing the parameters $\eta$ and $r_{c}$. Figures.5(a) and $5(\mathrm{~b})$ show the double-peaked structure of the $Q$-function. We see that how the separation between the two components can be controlled by varying the parameters $\eta$ and $r_{c}$. In other words, by varying $\eta$ and $r_{c}$ one can control the macroscopic distinguishability of the states involved in the superposition. Fig.5(c) shows an interesting case in which the membrane's state is a superposition of two different kind of states with equal amplitude, one of them is a coherent like state and another is a squeezed like state. We mention that this state can only be prepared for large values of $\eta=0.98$ and $r_{c}=0.998$.

\section{B. Nonlinear Schrödinger-like cat state}

In this subsection we consider the generation of the so-called nonlinear Schrödinger cat state (NLSCS) of the membrane. For this purpose, we assume that the membrane is initially prepared in the NLCS discussed in section. III, i.e., $|\psi(0)\rangle_{m}=|\zeta ; f\rangle_{m}=\sum_{k} C_{k}|k\rangle_{m}$ with $C_{k}=\aleph^{\prime} \frac{\zeta^{k} P(k)}{\sqrt{k !}}$, where the normalization constant is given 
by $\aleph^{\prime}=\left[\frac{|\zeta|^{2 k} P(k)^{2}}{k !}\right]^{-\frac{1}{2}}$. Thus the time dependent state of the system given by Eq. 20 at time $\tau=2 \pi$ reads

$|\psi(\tau=2 \pi)\rangle=\aleph^{\prime} \sum_{n, k} D_{n} e^{2 \pi i n^{2}|\beta|^{2} g(k)} \frac{\zeta^{k} P(k)}{\sqrt{k !}}|n\rangle_{f} \otimes|k\rangle_{m}$.
It should be noted that the experimental realization of the system under consideration shows $\eta<1$ and $\theta<<$ 1, (e,g. for the experimental values given in Refs. 21 23], we obtain $10^{-2} \lesssim \eta<1$ and $\left.\theta \lesssim 10^{-3}\right)$. Therefore, one may keep terms up to first order in the phonon number $\hat{n}_{b}$ in Eq. (8) and approximate the nonlinearity function $f_{(j=1)}\left(n_{b}\right)=f\left(n_{b}\right)$ by expanding the associated Laguerre polynomial

$$
f\left(\hat{n}_{b}\right) \simeq \epsilon+\sigma \hat{n}_{b}
$$

where

$$
\begin{array}{r}
\epsilon=\sum_{m=1} \sum_{k=0}^{\frac{m-1}{2}}(m-2 k) \frac{\left|r_{c}\right|^{m}}{m}\left(\begin{array}{c}
m \\
k
\end{array}\right) \frac{(m-1) !}{4^{m-1}\left[\left(\frac{m-1}{2}\right) !\right]^{2}}\left\{e^{-\frac{1}{2}(\eta \theta)^{2}(m-2 k)^{2}}\right\}, \\
\sigma=\sum_{m=1} \sum_{k=0}^{\frac{m-1}{2}} \frac{(i \eta \theta)^{2}(m-2 k)^{3}}{2 !} \frac{\left|r_{c}\right|^{m}}{m}\left(\begin{array}{c}
m \\
k
\end{array}\right) \frac{(m-1) !}{4^{m-1}\left[\left(\frac{m-1}{2}\right) !\right]^{2}}\left\{e^{-\frac{1}{2}(\eta \theta)^{2}(m-2 k)^{2}}\right\} .
\end{array}
$$

Under this approximation, the nonlinear function $g\left(n_{b}\right)$ reduces to

$$
g(k) \simeq \Gamma k^{2}-\Delta k+\epsilon^{2},
$$

where we have defined

$$
\Gamma=(\epsilon-\sigma)^{2}+\sigma \epsilon+2 \sigma ; \Delta=\sigma(\sigma-3 \epsilon)+\epsilon^{2} .
$$

By using Eq. (47) in Eq.(44), and assuming that the cavity field is initially prepared in the Fock state $|l\rangle$, $D_{n}=\delta_{n, l}$, the state of the membrane is obtained as

$$
|\psi(2 \pi)\rangle_{m}=\aleph^{\prime} e^{2 \pi i(l \epsilon|\beta|)^{2}} \sum_{k} e^{2 \pi i \xi k^{2}} \frac{\left(e^{i \varphi} \zeta\right)^{k} P(k)}{\sqrt{k !}}|k\rangle_{m},
$$

where

$$
\phi=2 \pi l^{2}|\beta|^{2} \Delta, \quad \xi=l^{2}|\beta|^{2} \Gamma .
$$

Depending on the value of the parameter $\xi$, the state $|\psi(2 \pi)\rangle_{m}$ can be made equivalent to a variety of nonlinear multicomponent cat states. As an example for, $\xi=0.25$ we have

$|\psi(2 \pi)\rangle_{m}=e^{2 \pi i(l \epsilon|\beta|)^{2}} \aleph_{+}\left(e^{i \pi / 4}|\zeta ; f\rangle_{m}+e^{-i \pi / 4}|-\zeta ; f\rangle_{m}\right)$.

where $\aleph_{+}=\left[2+2 \aleph^{\prime 2} \sum_{n=0} \frac{\left(-\zeta^{2}\right)^{n} P(n)^{2}}{n !}\right]^{-\frac{1}{2}}$ is the normalization constant 48 . The state (51) shows a twocomponent NLSCS. The all higher order-multicomponent nonlinear cats states of the motional state of the membrane may be obtained at time $\tau=2 \pi$ by varying the parameters $\xi$ and $\zeta$. The normalized $Q$-functions $(Q(\tau) \equiv$ $Q_{m}(\tau) / \aleph_{+}^{2}$ ) of the cat states produced by different values of $\xi$ and $\zeta$ and for a given value of the membrane's reflectivity are shown in Fig.6. It is interesting to note that by varying the membrane's reflectivity one can control the separation between the components of the generated NLSCS. To show this, we have plotted the normalized $Q$-function for three different values of $r_{c}$ in Fig.7. Obviously, by increasing the parameter $r_{c}$, the separation between the components of the produced NLSCS is increased.

\section{EFFECTS OF CAVITY FIELD DAMPING ON THE MOTIONAL STATES OF THE MEMBRANE}

In order to make the model under consideration more realistic, we now take into account the cavity field damping to examine its influence on the generated motional states of the membrane. For this purpose, we consider a regime in which the radiation mode relaxes much faster than the mirror. Experimentally, in the system under consideration the membrane Quality-factor is too large $\left(Q_{m}=10^{6}[21\right.$, 22] $)$ which leads to small membrane damping rate compare to the cavity damping $\kappa$. The case in which the mirror relaxes much faster than the cavity mode, does not show any quantum features due to the thermalization effects 28. We assume that the number of thermal photons is negligible at optical frequencies. Hence, the master equation for the whole system reads as 28 .

$$
\hat{\dot{\rho}}(t)=\frac{i}{\hbar}[\hat{\rho}, \hat{H}]+L(\hat{\rho}),
$$

with

$$
L(\hat{\rho})=\kappa / 2\left(2 \hat{a} \hat{\rho} \hat{a}^{\dagger}-\hat{a}^{\dagger} \hat{a} \hat{\rho}-\hat{\rho} \hat{a}^{\dagger} \hat{a}\right)
$$


where $\kappa$ denotes the rate of damping due to the photon leakage from the cavity. In order to solve Eq. (52) we introduce the new density operator $\hat{R}$ as $\hat{\rho}(\tau)=$ $\hat{U}(\tau) \hat{R} \hat{U}^{\dagger}(\tau)$. Here, $\hat{U}(\tau)$ is given by Eq. 16. By using this definition we rewrite the Eq. (52) as

$$
\hat{\dot{R}}=\hat{U}^{\dagger} L\left(\hat{U} \hat{R} \hat{U}^{\dagger}\right) \hat{U}^{\dagger}=\tilde{L}(\hat{R}) .
$$

We now assume the above differential equation has a solution as $\hat{R}=\hat{R}_{0}+\hat{R}_{1}$, where $\hat{R}_{0}$ is a time independent operator, i.e., $\hat{\dot{R}}_{0}=0$ (this operator corresponds to the free solution of Eq.(52)). While, $\hat{R}_{1}$ satisfies the following time dependent differential equation

$$
\hat{\dot{R}}_{1}=\tilde{L}\left(\hat{R}_{0}+\hat{R}_{1}\right) .
$$

Since the damping term $L(\hat{\rho})$ is small enough, one can apply the first order Born approximation to replace $\tilde{L}\left(\hat{R}_{0}+\hat{R}_{1}\right)$ by $\tilde{L}\left(\hat{R}_{0}\right)$ in the right-hand side of Eq. $(55)$. Under this approximation, the following solution for the operator $\hat{R}$ is obtained

$$
\hat{R}(\tau)=\hat{R}_{0}+\int_{0}^{\tau} \tilde{L}\left(\hat{R}_{0} ; t\right) d t
$$

Hence, the density matrix $\rho(t)$ is given by

$$
\hat{\rho}(\tau)=\hat{\rho}_{0}(\tau)+\hat{\rho}_{1}(\tau),
$$

where

$$
\hat{\rho}_{0}(\tau)=\hat{U}(\tau) \hat{\rho}(0) \hat{U}^{\dagger}(\tau),
$$

is the undamped part of the density matrix given by Eq. (26) and the damping part $\rho_{1}(\tau)$ has the following form

$$
\hat{\rho}_{1}(\tau)=\hat{U}(t)\left[\int_{0}^{\tau} \tilde{L}\left(\hat{R}_{0} ; t\right) d t\right] \hat{U}^{\dagger}(\tau) .
$$

To study the time evolution of the system in the presence of cavity damping we turn our attention to the density matrix of Eq. 26) in which the cavity field is assumed to be initially in a coherent superposition of Fock states, and the membrane is assumed to be initially prepared in the motional ground state. By using Eq.(53) the damping part $\hat{\rho}_{1}(\tau)$ reads as

$$
\begin{aligned}
\hat{\rho}_{1}(\tau)= & \kappa \int_{0}^{\tau}\left[\tilde{\hat{a}}(\tau-t) \hat{\rho}_{0}(\tau) \tilde{\hat{a}}^{\dagger}(\tau-t)\right] d t \\
& -\kappa / 2\left[\hat{a}^{\dagger} \hat{a} \hat{\rho}_{0}(\tau)+\hat{\rho}_{0}(\tau) \hat{a}^{\dagger} \hat{a}\right],
\end{aligned}
$$

where we have defined

$$
\tilde{\hat{a}}(\tau-t)=\hat{U}(\tau-t) \hat{a} \hat{U}^{\dagger}(\tau-t) .
$$

Substituting from Eq.(B2) into Eq.(61) and applying the Baker-Campbell-Hausdorff formula together with using the Feynman disentangling theorem we arrive at

$$
\tilde{\hat{a}}(\tau-t)=\hat{a} e^{\beta \mu(\tau-t) \hat{B}^{\dagger}} e^{-\beta^{*} \mu^{*}(\tau-t) \hat{B}} e^{i|\beta|^{2} \lambda(\tau-t) g\left(\hat{n}_{b}\right)} .
$$

By substituting $\hat{\rho}_{0}(\tau)$ from Eq. (26) into Eqs. 60 we readily obtain

$$
\begin{aligned}
\hat{\rho}_{1}(\tau)= & \kappa \sum_{n, l} D_{n} D_{l}^{*} e^{i\left(n^{2} \Theta-l^{2} \Theta^{*}\right) g(0)} \int_{0}^{\tau}\left[\left(\tilde{\hat{a}}(\tau-t)|n\rangle_{f} \otimes\left|\Lambda_{n}(\tau)\right\rangle_{m}\right)\left({ }_{f}\langle l| \otimes{ }_{m}\left\langle\Lambda_{l}(\tau)\right| \tilde{\hat{a}}^{\dagger}(\tau-t)\right)\right] d t \\
& -\kappa / 2\left[\sum_{n, l}(n+l) D_{n} D_{l}^{*} e^{i\left(n^{2} \Theta-l^{2} \Theta^{*}\right) g(0)}|n\rangle_{f f}\left\langle l|\otimes| \Lambda_{n, 0}(\tau)\right\rangle_{m m}\left\langle\Lambda_{l, 0}(\tau)\right|\right] .
\end{aligned}
$$

Finally by using Eq. 62 we find the following expression for $\hat{\rho}_{1}(\tau)$

$$
\begin{aligned}
\hat{\rho}_{1}(\tau)= & \kappa \sum_{n, l} \sum_{k, k^{\prime}} D_{n} D_{l}^{*} I_{n, l}^{k, k^{\prime}}(\tau) \int_{0}^{\tau}\left[e^{i|\beta|^{2} \lambda(\tau-t) g(k)} e^{-i|\beta|^{2} \lambda^{*}(\tau-t) g\left(k^{\prime}\right)}|n-1\rangle_{f f}\left\langle l-1|\otimes| \Lambda_{1, k}(\tau-t)\right\rangle_{m m}\left\langle\Lambda_{1, k^{\prime}}(\tau-t)\right|\right] d t \\
& -\kappa / 2\left[\sum_{n, l}(n+l) D_{n} D_{l}^{*} e^{i\left(n^{2} \Theta-l^{2} \Theta^{*}\right) g(0)}|n\rangle_{f f}\left\langle l|\otimes| \Lambda_{n, 0}(\tau)\right\rangle_{m m}\left\langle\Lambda_{l, 0}(\tau)\right|\right]
\end{aligned}
$$

where by definition

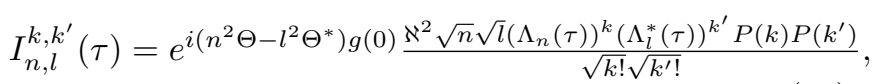


We are now in a position to examine the influence of the cavity field damping on the motional state of the membrane by using the density matrix of the Eq. (57). As before, we assume the membrane to be prepared in a vibrational vacuum state initially. In Fig. 8 we have plotted the time evolution of the Mandel parameter of the membrane motional state for various values of the rate of cavity field damping, the membrane's reflectivity and the Lamb- Dicke parameter. From Fig.8(a) it is evident that unlike the undamped case $(\kappa=0)$, at the initial stages of evolution the Mandel parameter increases which shows super-Poissonian statistics. However, as time goes on, this parameter decreases and the sub-Poissonian statistics occurs. The rate with which the enhancement of super-Poissonian (sub-Poissonian) statistics occurs is directly proportional to the membrane's reflectivity $r_{c}$; the greater $r_{c}$ is, the more quickly the Mandel parameter increases (decreases). Furthermore, as in the undamped case, with the increasing value of $r_{c}$ the sub-Poissonian characteristic of the motional state of the membrane is strengthened. Also, Fig.8(b) shows that by increasing the lamb-Dicke parameter $\eta$ the sub-Poissonian behaviour is enhanced. In Fig.9 we have illustrated the time evolution of the squeezing parameter $S_{2}(\tau)$ in the presence of the cavity field damping. As is seen, the photon leakage from the cavity suppresses the quadrature squeezing of the motional state of the membrane in the course of time evolution. However, it is evident that increasing the Lamb-Dicke parameter $\eta$ (Fig.9a) or the membrane's reflectivity $r_{c}$ (Fig.9b) brings about noise reduction in the quadrature $X_{2}$ and thus will increase the quadrature squeezing. Thus, we conclude that the destructive effect of the cavity field damping on the nonclassicality associated with the motional state of the membrane can effectively be controlled by changing the parameters $r_{c}$ and $\eta$.

As another aspect of the influence of the cavity field damping on motional state of the membrane, we turn our attention to the NLSCS introduced in Sec.IV. For this purpose, we consider the density matrix of Eq.(57) at time $\tau=2 \pi$ in which $\hat{\rho}_{0}(2 \pi)$ corresponds to the nonlinear multicomponent state of Eq.49) and $\hat{\rho}_{1}(2 \pi)$ is obtained from Eq. (57). As an example, the corresponding normalized quasiprobability $Q$-function $\left(Q_{m}(\tau) / \aleph^{2}\right)$ at $\tau=2 \pi$ for two different values of the cavity field damping rate $\kappa$ and for given values of the parameters $r_{c}, \xi, \zeta$ is illustrated in Fig.10. As is seen, for small value of $\kappa$ the $Q$ function consists of two well-separated peaks (Fig.10(a)). By increasing the damping rate, one not only decreases the height of the two peaks but also decreases the spatial separation between the NLCS components involved in the superposition (Fig. 10(b)). In this connection, further numerical analysis reveals that by changing the parameters $r_{c}$ and $\eta$ one can control the spatial separation between the components of the generated NLSCS (i.e., controlling the influence of decoherence). It is also worth noting that the generated motional NLSCS preserves more or less its quantum coherence even in the presence of the cavity field damping. This survival of quantum coherence, which can be attributed to the nonlinear character of the system under consideration, is in full agreement with the fact that deformed (nonlinear) coherent states superposition can be more robust against decoherence than the usual (nondeformed) Schrödinger cat states [48. It has been shown [36 that deformed states, due to their nonlinear character, give rise to a more rich phase space structure, part of which can easier survive against decoherence.

\section{SUMMARY AND CONCLUSIONS}

In this paper we have introduced a physical scheme that allows one to generate and control the nonclassical properties of motional nonlinear coherent states and their superpositions for an undamped vibrating micromechanical membrane inside an optical cavity. We have shown that if the cavity field is initially prepared in a Fock state the motional state of the membrane may evolve to a family of nonlinear coherent states. We have been interested in analyzing the nonclassical properties of the generated state of the membrane, including the quadrature squeezing and the sub-Poissonian statistics. In particular, we have found that the Lamb-Dicke parameter and the membrane's reflectivity lead to an enhancement of the nonclassical properties. As we have seen, with increasing the Lamb-Dicke parameter and the membrane's reflectivity the sub-Poissonian behaviour and quadrature squeezing of the motional state of the membrane are considerably strengthend. In addition, the scheme offers the possibility of generating various types of the so-called nonlinear multicomponent Schrödinger cat sates of the membrane. We have shown that the separation between nonlinear coherent components is increased by increasing the parameters $\eta$ and $r_{c}$. We have also extended our treatment to a more realistic situation in which the photon leakage from the cavity as a relevant source of decoherence is included and have examined its influence on the nonclassical characteristics of the generated motional states of the membrane. We have shown that it is possible to control the effect of the cavity field damping on the nonclassical behaviour of the motional state of the membrane via the Lambe-Dicke parameter and the membrane's reflectivity. In particular, we have found that the generated motional NLSCSs of the membrane can be more robust against decoherence than the usual Schrödinger cat states.

At the end, we would like to point out that our treatment is restricted to the case of one-phonon excitations $(\mathrm{j}=1)$ of the vibrational sideband. It is expected to generate some other interesting nonclassical motional states of the membrane, e.g. nonlinear squeezed states, by considering higher order of excitations. We hope to report on this issue in a forthcoming paper. 


\section{ACKNOWLEDGMENTS}

The authors would like to express their gratitude to the referees, whose valuable comments have improved the paper. They are also grateful to the Office of Graduate
Studies of the University of Isfahan for their support.

\section{Appendix A: Nonlinearity function $f_{j}\left(\hat{n}_{b}\right)$}

By applying the Baker-Campbell-Hausdorff theorem in Eq.(3) to disentangling the exponential terms and by using the series expansion of each exponential term we obtain

$$
\omega_{c}(\hat{x})=\frac{\pi c}{2 L}-\frac{c}{2 L} \sum_{m=1}^{\infty} \sum_{k=0}^{\frac{m-1}{2}} \frac{\left|r_{c}\right|^{m}}{m}\left(\begin{array}{c}
m \\
k
\end{array}\right) \frac{(m-1) !}{4^{m-1}\left[\left(\frac{m-1}{2}\right) !\right]^{2}} e^{-\frac{1}{2}(\eta \theta)^{2}(m-2 k)^{2}} \sum_{l=0}\left\{[(i \eta \theta)(m-2 k)]^{2 l+j} \frac{\left(\hat{b}^{\dagger}\right)^{l} \hat{b}^{l}}{l !(l+j) !} \hat{b}^{j}+h . c .\right\} .
$$

By making use of the relation

$$
\left(\hat{b}^{\dagger}\right)^{l} \hat{b}^{l}=\frac{\hat{n}_{b} !}{\left(\hat{n}_{b}-l\right) !},
$$

Eq. A1 can be written as

$$
\omega_{c}(\hat{x})=\frac{\pi c}{2 L}-\frac{c}{2 L}(i \eta \theta)^{j}\left\{f_{j}\left(\hat{n}_{b}\right) \hat{b}^{j}+h . c .\right\}
$$

where

$$
f_{j}\left(\hat{n}_{b}\right)=\sum_{m=1} \sum_{k=0}^{\frac{m-1}{2}} \frac{\left|r_{c}\right|^{m}}{m}\left(\begin{array}{c}
m \\
k
\end{array}\right) \frac{(m-1) !}{4^{m-1}\left[\left(\frac{m-1}{2}\right) !\right]^{2}}\left\{e^{-\frac{1}{2}(\eta \theta)^{2}(m-2 k)^{2}}\right\} \frac{\hat{n}_{b} !(m-2 k)^{j}}{\left(\hat{n}_{b}+j\right) !}\left[\sum_{l=0} \frac{\left[-(\eta \theta)^{2}(m-2 k)^{2}\right]^{l}}{l !(l+j) !} \frac{\left(\hat{n}_{b}+j\right) !}{\left(\hat{n}_{b}-l\right) !}\right] .
$$

Rewriting the above equation in terms of the associated Laguerre polynomials, $L_{n}^{k}(v)=\sum_{m} \frac{(-v)^{m}(n+k) !}{(n-m) !(k+m) ! m !}$, leads to Eq.8.

\section{Appendix B: Disentangling of the time evolution operator by using Feynman operator calculus}

The unitary time evolution operator for the case of one-photon excitations $(j=1)$ can be written as

$$
\hat{U}^{(j=1)}(t)=e^{-i \omega_{0} \hat{a}^{\dagger} \hat{a} t} \hat{U}_{i n t}^{(j=1)}(t),
$$

where

$$
\hat{U}_{i n t}^{(j=1)}(t)=\hat{T} \exp \left(-i \hat{a}^{\dagger} \hat{a} \int_{0}^{t}\left(g \hat{B}^{\dagger} e^{i \omega_{m} s}+g^{*} \hat{B} e^{-i \omega_{m} s}\right) d s\right)
$$

with $\hat{B}=f(\hat{n}) \hat{b}$ as the nonlinear(deformed) annihilation operator obeying the following commutation relation

$$
\left[\hat{B}, \hat{B}^{\dagger}\right]=(\hat{n}+1) f^{2}(\hat{n})-\hat{n} f^{2}(\hat{n}-1) .
$$

By using the Feynman operator calculus 46 we readily obtain

$$
\hat{U}_{i n t}^{(j=1)}(t)=e^{\beta \mu(t) \hat{a}^{\dagger} \hat{a} \hat{B}^{\dagger}} \exp \left[-i g^{*} \hat{a}^{\dagger} \hat{a} \int_{0}^{t} \hat{B}^{\prime}(s) e^{-i \omega_{m} s} d s\right],
$$

where

$$
\hat{B}^{\prime}(s)(s)=e^{-\beta \mu(s) \hat{a}^{\dagger} \hat{a} \hat{B}^{\dagger}} \hat{B} e^{\beta \mu(s) \hat{a}^{\dagger} \hat{a} \hat{B}^{\dagger}} .
$$

By applying the Baker-Campbell-Hausdorff formula together with the commutation relation (B3), Eq. B5) can be written as

$$
\hat{B}^{\prime}(s)(s)=\hat{B}+\sum_{m=1}^{\infty} \frac{[-k(s)]^{m}}{m !} \hat{B}^{\dagger^{m-1}} \sum_{i=0}^{m}(-1)^{i} \frac{m !}{i !(m-i) !} F(\hat{n}+i),
$$

where $k(s)=\beta \mu(s) \hat{a}^{\dagger} \hat{a}$ and $F(\hat{n})=\hat{n} f^{2}(\hat{n}-1)$. Finally, by using the Feynman disentangling theorem we arrive at

$$
\begin{array}{r}
\hat{U}_{i n t}^{(j=1)}(\tau)=e^{\beta \mu(\tau) \hat{a}^{\dagger} \hat{a} \hat{B}^{\dagger}} e^{-\beta^{*} \mu^{*}(\tau) \hat{a}^{\dagger} \hat{a} \hat{B}} \times \\
e^{i|\beta|^{2} \lambda(\tau)\left(\hat{a}^{\dagger} \hat{a}\right)^{2} g\left(\hat{n}_{b}\right)} e^{O\left(\beta^{3}, \hat{n}_{b}\right)+\ldots}
\end{array}
$$


[3] T.J. Kippenberg and K. J. Vahala, Opt.Express 15, 17172(2007).

[4] C. Fabre, M. Pinard, S. Bourzeix, A. Heidmann, E. Giacobino, and S. Reynaud, Phys. Rev. A 49, 1337 (1994); K. Jacobs, P. Tombesi, M. J. Collett, and D. F. Walls, Phys. Rev. A 49, 1961 (1994).

[5] V. B. Braginsky and F. Y. Khalili, Quantum Measurement(Cambridge University Press, Cambridge, 1992).

[6] A. Abramovici et al., Science 256, 325(1992).

[7] D. Rugar and P. Hansma, Phys. Today 43(10), 23(1990).

[8] J. Zhang, K. Peng, and S. L. Braunstein, Phys. Rev. A 68, 013808(2003).

[9] D. Kleckner and D. Bouwmeester, Nature (London) 444, 75 (2006).

[10] S. Gigan et al., Nature (London) 444, 67 (2006).

[11] M. Bhattacharya, H. Uys, and P. Meystre, Phys. Rev. A 77, 033819 (2008).

[12] P. F. Cohadon, A. Heidmann, and M. Pinard, Phys. Rev. Lett. 83, 3174(1999); Y. Hadjar et al., Europhys. Lett. 47, 545(1999); S. Mancini, V. Giovannetti, D. Vitali, and P. Tombesi, Phys. Rev. Lett. 88, 120401(2002); C. H. Metzger and K. Karrai, Nature (London) 432, 1002 (2004); B. S. Sheard et al., Phys. Rev. A 69, 051801(R)(2004); H. Rokhsari, T.J. Kippenberg, T. Carmon, and K. J. Vahala, IEEE J. Quantum Electron. 12, 96(2006); O. Arcizet et al., Nature (London) 444, 71 (2006); A. Schliesser et al., Phys. Rev. Lett. 97, 243905 (2006); A. DiVirgilio et al., Phys. Rev. A 74, 013813(2006); M. Bhattacharya and P. Meystre, Phys. Rev. Lett. 99, 073601 (2007); M. Ludwig, B. Kubala and F. Marquardt, New. J. Phys. 10, 095013(2008); A. Xuereb et al., Phys. Rev. A 79, 053810(2009).

[13] W. Marshall, C. Simon, R. Penrose, and D. Bouwmeester, Phys. Rev. Lett. 91, 130401 (2003).

[14] Y. T. Yang et al., Nano Lett. 6, 583 (2006).

[15] T. Corbitt et al., Phys. Rev. Lett. 98, 150802 (2007).

[16] J. M. Courty, A. Heidmann, and M. Pinard, Phys. Rev. Lett. 90, 083601(2003).

[17] P. Treutlein, D. Hunger, S. Camerer, T. W. Hansch, and J. Reichel, Phys. Rev. Lett. 99, 140403 (2007).

[18] S. Mancini, D. Vitali, and P. Tombesi, Phys. Rev. Lett. 90, 137901(2003).

[19] C. Genes, H. Ritsch, and D. Vitali, Phys. Rev. A 80, 061803(R)(2009).

[20] S. Mancini, D. Vitali, V. Giovannetti, and P. Tombesi, Eur. Phys. J. D 22,417(2003).

[21] J. D. Thompson et al., Nature (London) 452, 72 (2008).

[22] A. M. Jayich et al., New. J. Phys. 10, 095008 (2008).

[23] M. Bhattacharya and P. Meystre, e-print arXiv: 0803.1219v1; J. C. Sankey, C. Yang, B. M. Zwickl, A. M. Jayich, and J. G. Harris, e-print arXiv:1002.4158.

[24] F. Helmer, M. Mariantoni, E. Solano, and F. Marquardt,Phys. Rev. A 79, 052115(2009); H. Miao, S. Danilishin, T. Corbitt, and Y. Chen, Phys. Rev. Lett. 103, 100402(2009); A. Clerk, F. Marquardt, and J. G. E. Harris, e-print arXiv:1002.3140

[25] A. Nunnenkamp, K. Børkje, J. G. E. Harris, and S. M. Girvin, e-print arXiv:1004.2510v2.

[26] M. Bhattacharya and P. Meystre, Phys. Rev. A 78, 041801(R)(2008).

[27] S. Bose, K. Jacobs, and P. L. Knight, Phys. Rev. A 56, 4175 (1997).

[28] S. Mancini, V. I. Manko, and P. Tombesi, Phys. Rev. A 55, 3042 (1997)
[29] A. F. Pace, M. J. Collett, and D. F. Walls, Phys. Rev. A 47, 3173 (1993); S. Mancini, D. Vitali, and P. Tombesi, Phys. Rev. Lett. 80,688 (1998); M. Pinard et al., Europhys. Lett. 72, 747(2005); T.J. Kippenberg and K. Vahala, Science 321, 1172(2008); I. Favero and k. Karrai, Nature Photon. 3, 201(2009); F. Marquardt and S. M. Girvin, Physics 2, 40(2009); O. RomeroIsart, M. L. Juan, R. Quidant, and J. I. Cirac, e-print arXiv:0909.1469v3.

[30] K. Jähne et al., Phys. Rev. A 79, 063819(2009).

[31] D. F. Walls, Nature(London) 306, 141(1983); R. E. Slusher et al., Phy. Rev. Lett. 56, 788(1986).

[32] S. B. Zheng, J. Opt. B: Quantum Semiclassical Opt. 10, 657 (1998).

[33] D. Kleckner et al., e-print arXiv:0807.1834 4.

[34] Zhang-qi Yin and Y. J. Han, e-print arXiv:0811.0424v3.

[35] A. Ferreira, A. Guerreiro, and V. Vedral, Phys. Rev. Lett. 96, 060407(2006); D. Vitali et al.,Phy. Rev. Lett. 98, 030405(2007).

[36] R. L. de Matos Filho and W. Vogel, Phys. Rev. A 54, 4560 (1996).

[37] V. I. Man'ko, G. Marmo, E. C. G. Sudarshan, and F. Zaccaria, Phys. Scr. 55, 528 (1997); V. I. Man'ko, G. Marmo, A. Porzio, S. Solimeno, and F. Zaccaria, Phys. Rev. A 62, 053407 (2000); B. Roy, P. Roy, J.Opt. B: Quantum and Semiclass. Opt. 2, 65(2000); S. Twareque Ali, R. Roknizadeh, and M. K. Tavassoly, J. Phys. A: Math. Gen. 37, 4407(2004).

[38] P. Aniello, V. I. Man'ko, G. Marmo, S. Solimeno, and F. Zaccaria, J. Opt. B: Quantum Semiclassical Opt. 2, $718(2000)$.

[39] S. Sivakumar, J. Opt. B: Quantum Semiclassical Opt. 2, R61(2000); B. Roy and P. Roy, J. Opt. B: Quantum Semiclassical Opt. 2, 65(2000); X. G. Wang, Opt. Commun, 178, 365(2000); J. Récamier and R. Jáuregui, J. Opt. B: Quantum Semiclassical Opt. 5, S365(2003); M. Abdel-Aty and A-S. F. Obada, Eur. Phy. J . D 23 155(2003); A-S. F. Obada and M. Darwish, J. Opt. B: Quantum Semiclassical Opt. 5 211(2003); A-S. F. Obada and G. M. Abd Al-Kader, J. Opt. B: Quantum Semiclassical Opt. 7 S635(2005); T. M. Duc and J. Noh, Opt. Commun. 281, 2842(2008).

[40] W. Vogel and R. L. de Matos Filho, Phys. Rev. A 52, 4214 (1995).

[41] A. Mahdifar, W. Vogel, Th. Richter, R. Roknizadeh, and M. H. Naderi, Phys. Rev. A 78, 063814 (2008).

[42] M. H. Naderi, M. Soltanolkotabi, and R. Roknizadeh,Eur. Phys. J. D 32, 397 (2005).

[43] K. Borkje et al.,e-print arXiv:1004.3587.

[44] I. Wilson-Rae et al.,New. J. Phys. 10, 095007 (2008).

[45] I. Wilson-Rae et al., Phys. Rev. Lett. 99, 093901 (2007).

[46] R. P. Feynman, Phys. Rev. 84, 108(1951).

[47] O. Kilic et al. Opt. Lett. 29, 2782(2004).

[48] S. Mancini and V. I. Man'ko, Europhys. Lett. 54, 586(2001). 


\section{FIGURE CAPTIONS}

Fig.1: A high-finesse optical cavity with two rigid end mirrors and a dielectric membrane centered at the middle of cavity.

Fig.2: The nonlinearity function $f\left(n_{b}\right)$ as a function of phonon number $n_{b}$ for: (a) $r_{c}=0.99, \eta=0.8$. (b) $r_{c}=0.9, \eta=10^{-5}$. Here we have set $\theta=10^{-4}$.

Fig.3: Time evolution of the squeezing parameter $S_{2}(\tau)$, corresponding to the generated NLCS $(23)$ versus the scaled time $\tau$ : (a) for $r_{c}=0.9$ and $\eta=0.14$ (black line), $\eta=0.19$ (blue line), and $\eta=0.24$ (green line). (b)for $r_{c}=0.98$ and $\eta=0.1$ (black line), $\eta=0.14$ (blue line), and $\eta=0.18$ (green line). Here we have set $L=0.07 \mathrm{~m}, m=50 \mathrm{pg}$.

Fig.4: Time evolution of the Mandel parameter $M(\tau)$ corresponding to the NLCS (23) as a function of $\tau$ : (a) $r_{c}=0.9$, (b) $r_{c}=0.98$ with $\eta=0.25$ (black line), and $\eta=0.3$ (red line).

Fig.5: The normalized $Q$-function $\left(Q(\tau) \equiv Q_{m}(\tau) / \aleph^{2}\right)$ of the generated state 41 of the membrane at time $\tau=2.9$ : (a) $r_{c}=0.95, \eta=0.8$; (b) $r_{c}=0.998, \eta=0.82$; (c) $r_{c}=0.998, \eta=0.98$.

Fig.6: The normalized $Q$-function $\left(Q(\tau) \equiv Q_{m}(\tau) / \aleph_{+}^{2}\right)$ of the multi-component cat states of the membrane at time $\tau=2 \pi$ for different values of $\xi$ and $\zeta$, and for $r_{c}=0.95:(\mathrm{a}) \xi=1.1, \zeta=0.25 ;(\mathrm{b}) \xi=1.8, \zeta=0.25$; (c) $\xi=1.8, \zeta=\sqrt{\frac{1}{6}}$.

Fig.7: The normalized $Q$-function $\left(Q(\tau) \equiv Q_{m}(\tau) / \aleph_{+}^{2}\right)$ of the multi-component cat states of the membrane at time $\tau=2 \pi$ for different values of the membrane's reflectivity $r_{c}$, and for $\xi=1 / \sqrt{8}, \zeta=1.8$ : (a) $r_{c}=0.8$. (b) $r_{c}=0.87$. (c) $r_{c}=0.99$.

Fig.8: Time evolution of the Mandel parameter $M(\tau)$ for different values of the cavity damping rate: (a) $\eta=0.19$, and $r_{c}=0.93, \kappa=0$ (black line), $r_{c}=0.93, \kappa=1 \omega_{m}$ (green line), $r_{c}=0.95, \kappa=1 \omega_{m}$ (blue line); (b) $r_{c}=0.95$ and $\eta=0.19, \kappa=0$ (black line), $\eta=0.19, \kappa=1 \omega_{m}$ (blue line), $\eta=0.16, \kappa=1 \omega_{m}$ (red line).

Fig.9: Time evolution of the squeezing parameter $S_{2}(\tau)$ for different values of the cavity damping rate: (a) $r_{c}=0.95$ and $\eta=0.19, \kappa=0$ (black line), $\eta=0.19, \kappa=1 \omega_{m}$ (blue line), $\eta=0.16, \kappa=1 \omega_{m}$ (red line); (b) $\eta=0.16$, and $r_{c}=0.96, \kappa=0$ (black line), $r_{c}=0.96, \kappa=1 \omega_{m}$ (blue line), $r_{c}=0.95, \kappa=1 \omega_{m}$ (red line).

Fig.10: The normalized $Q$-function of the multi- component cat states of the membrane at time $\tau=2 \pi$ in the presence of cavity field damping for two different values of the damping rate $\kappa$, and for $r_{c}=0.95$, $\xi=1.8, \zeta=0.25:(\mathrm{a}) \kappa=0.01 \omega_{m},(\mathrm{~b}) \kappa=0.4 \omega_{m}$. 


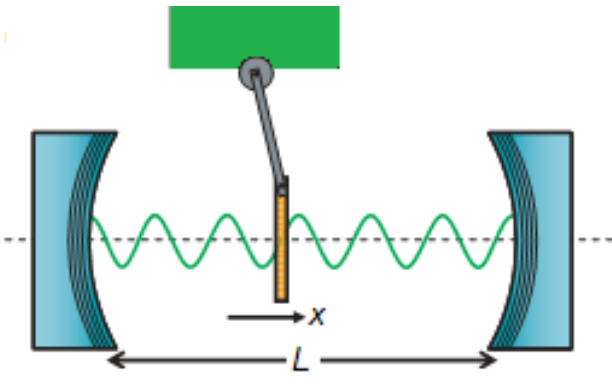

FIG. 1.

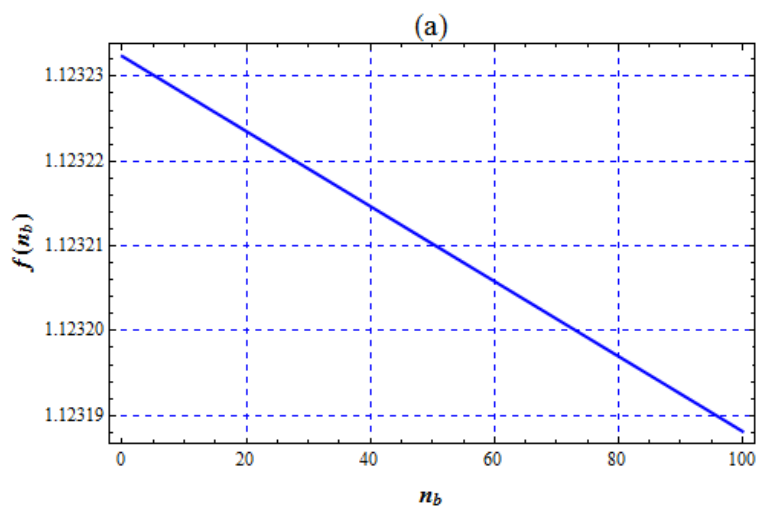

(b)

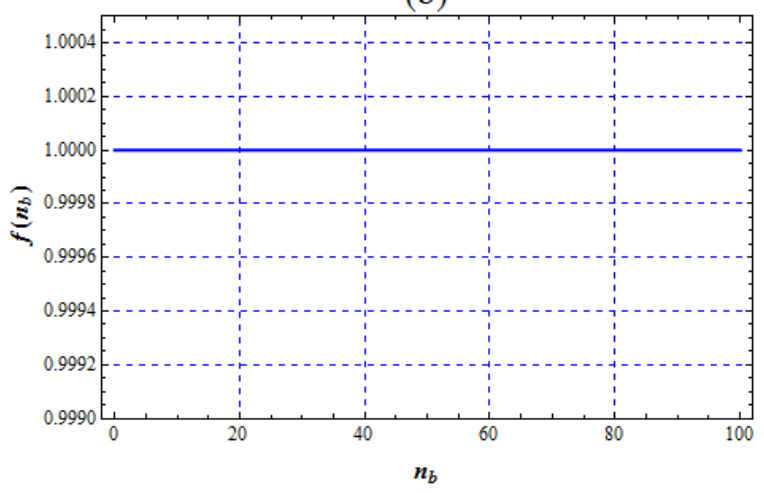

FIG. 2. 

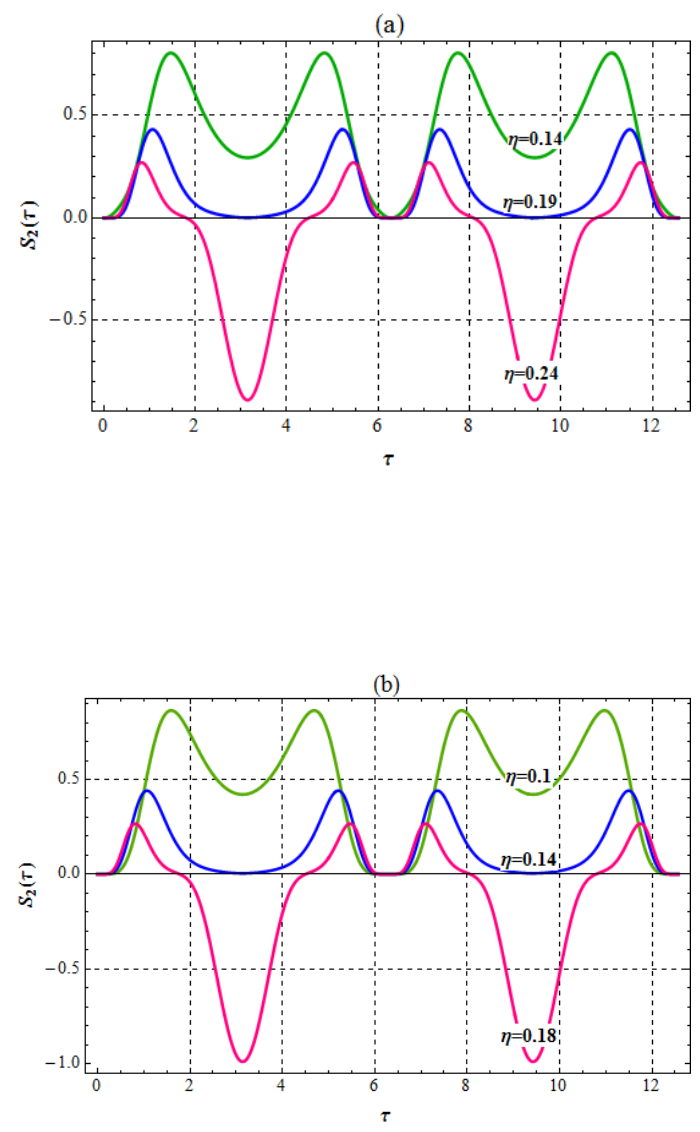

FIG. 3. 

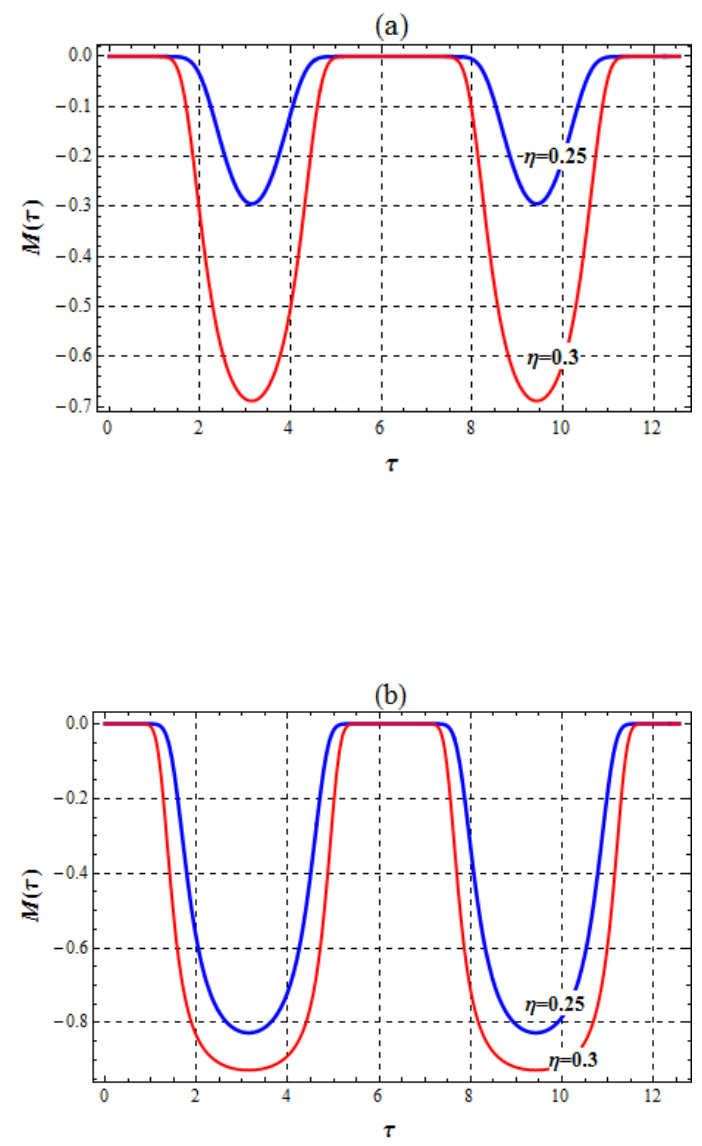

FIG. 4. 


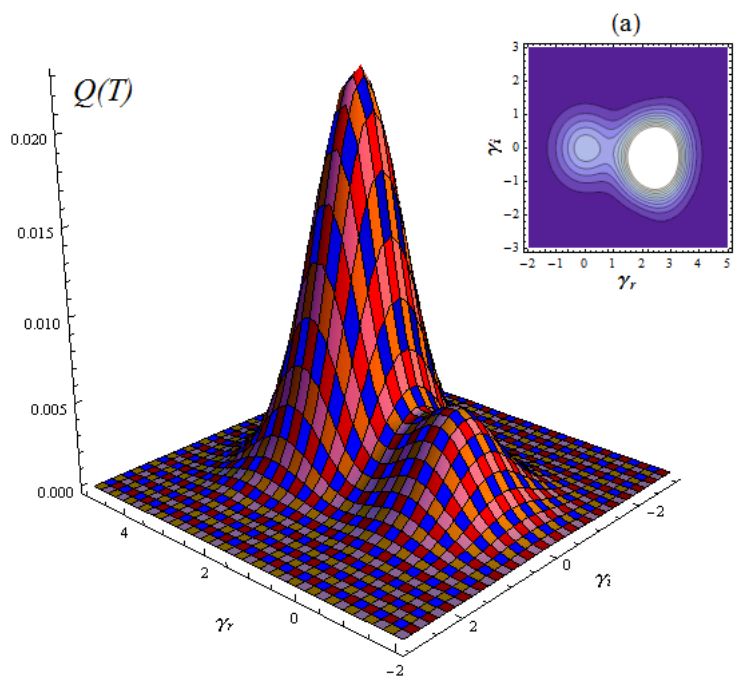

(b)

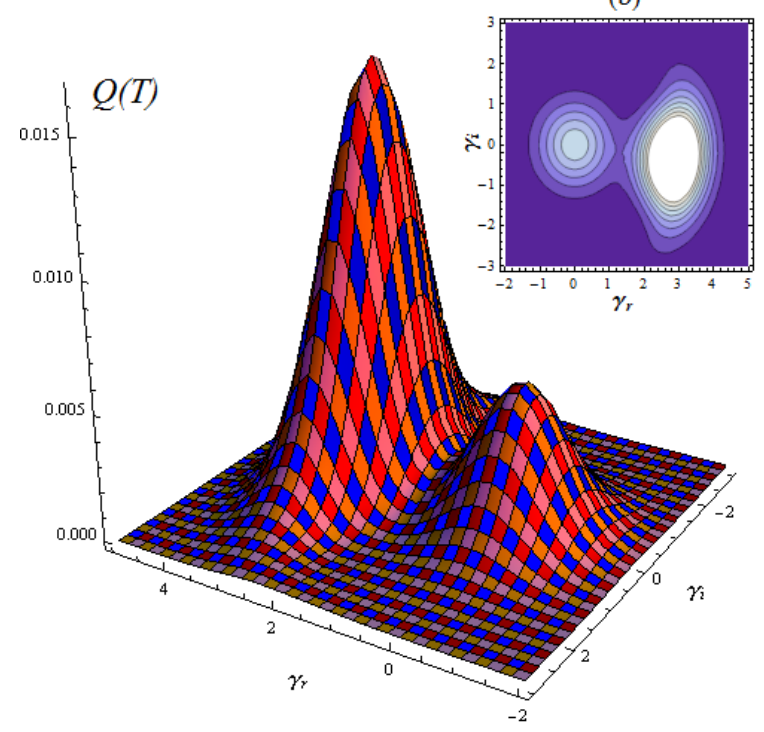

(c)

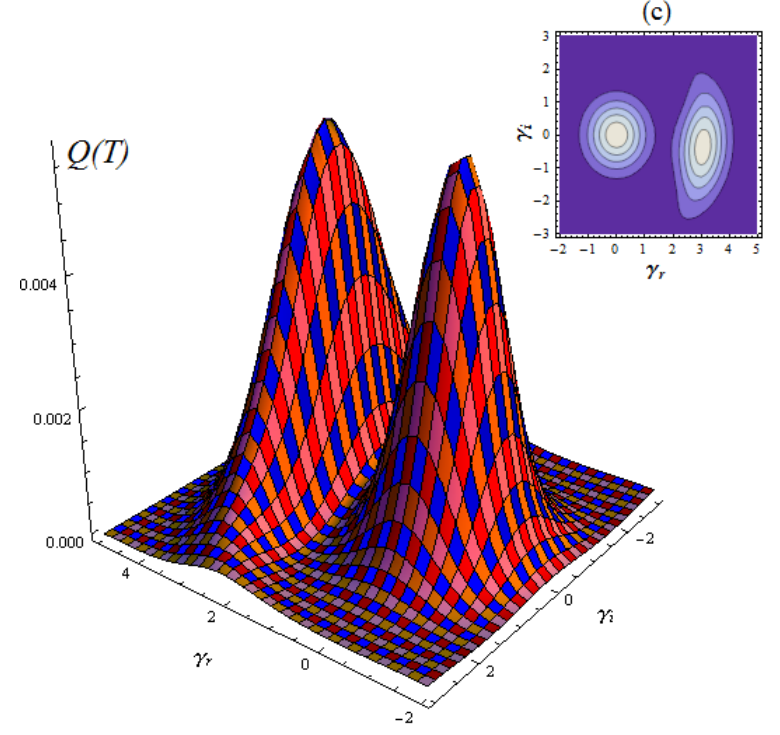

FIG. 5. 

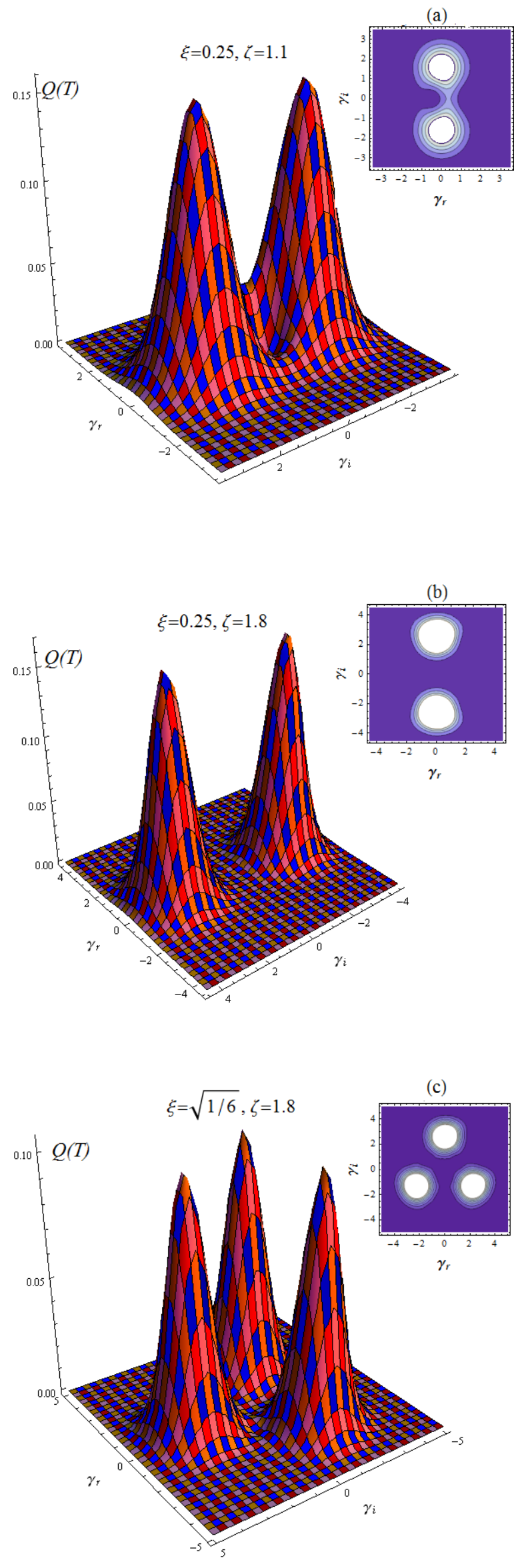

FIG. 6. 

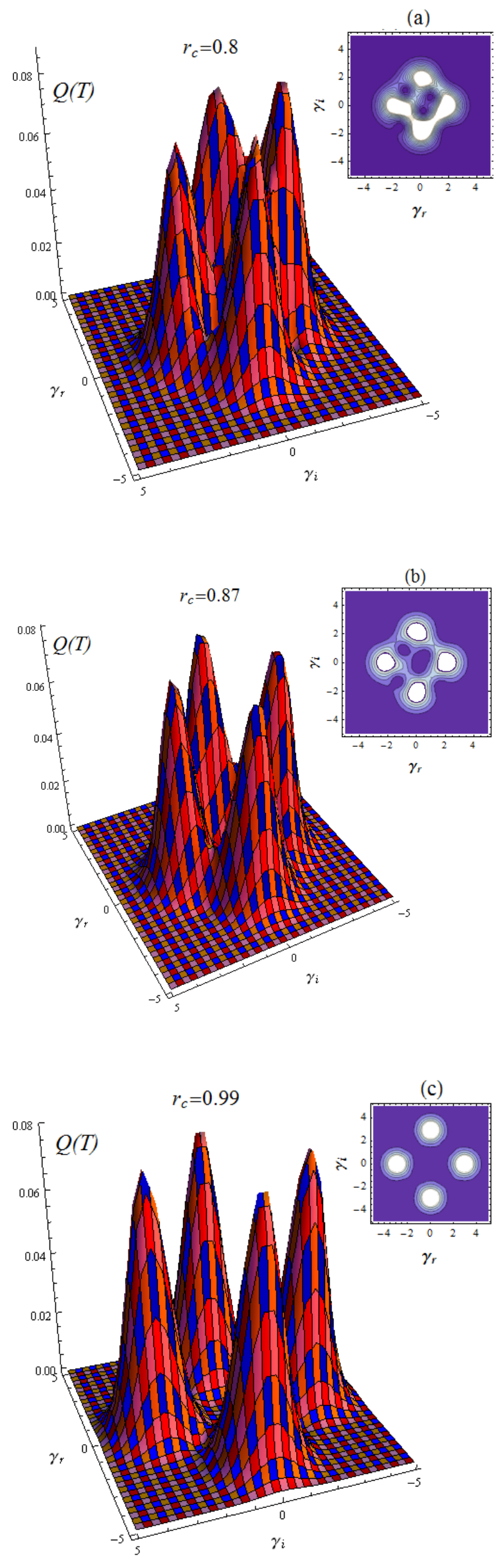

FIG. 7. 

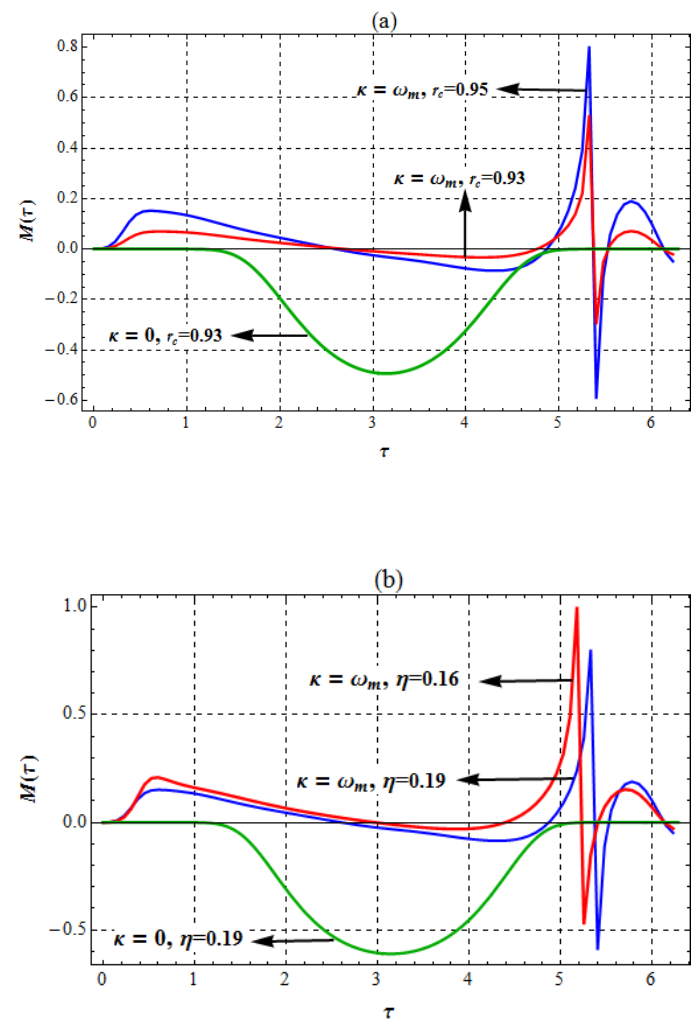

FIG. 8.
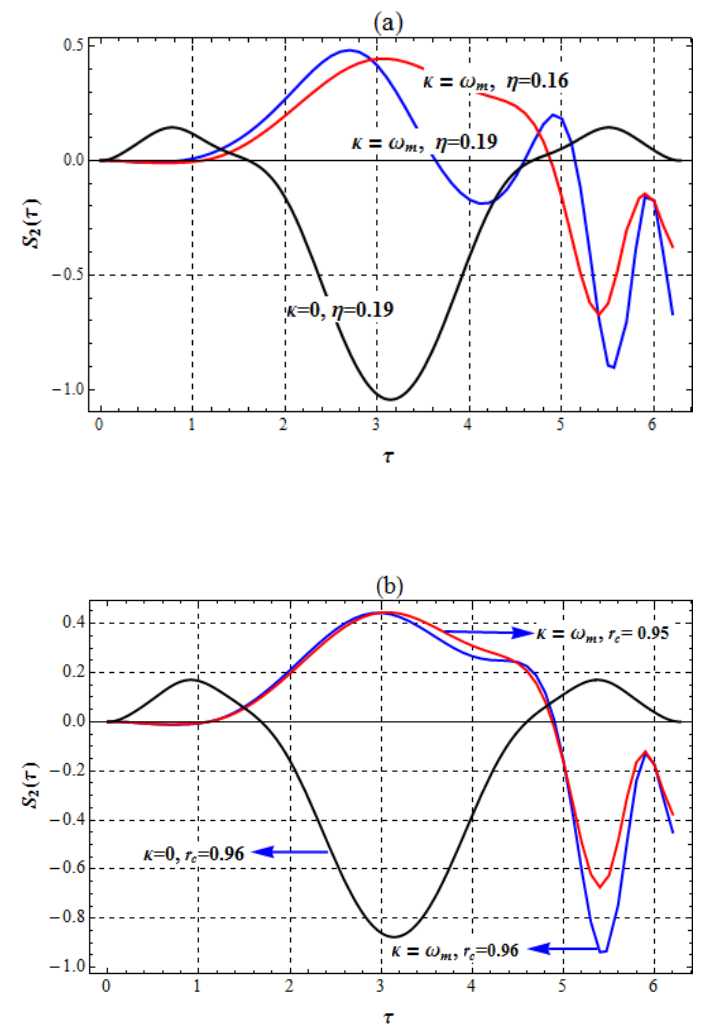

FIG. 9. 

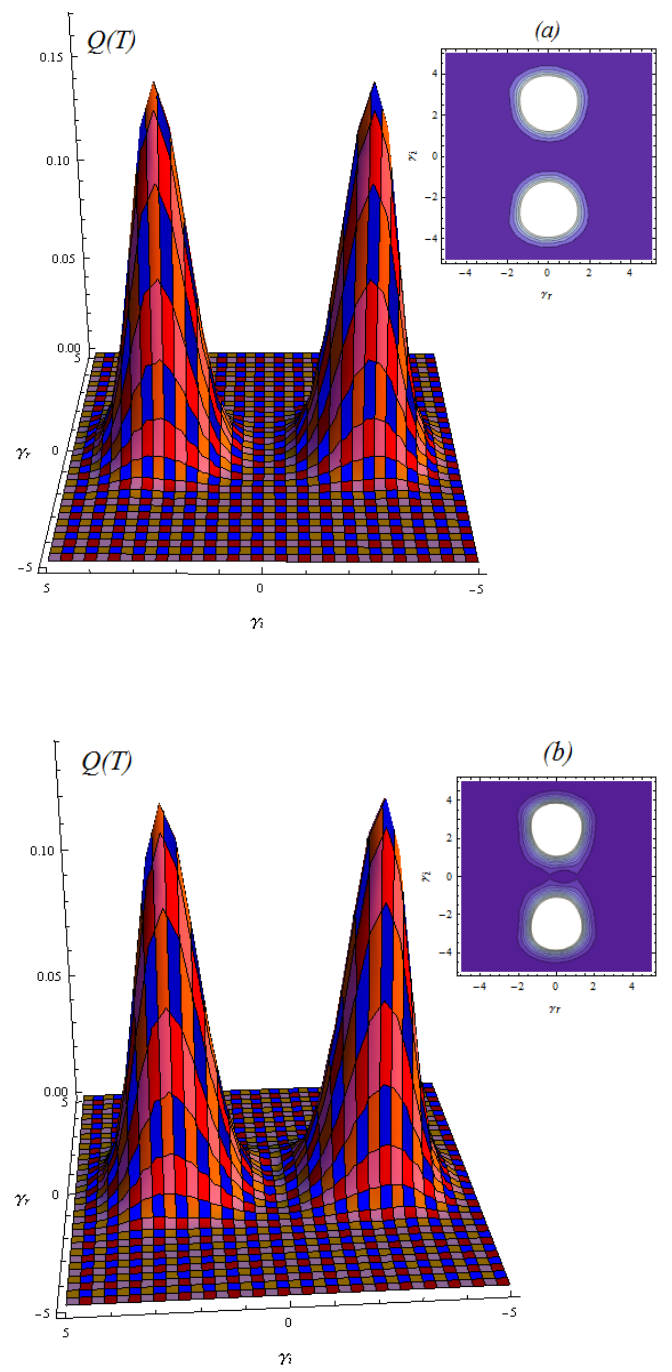

FIG. 10. 